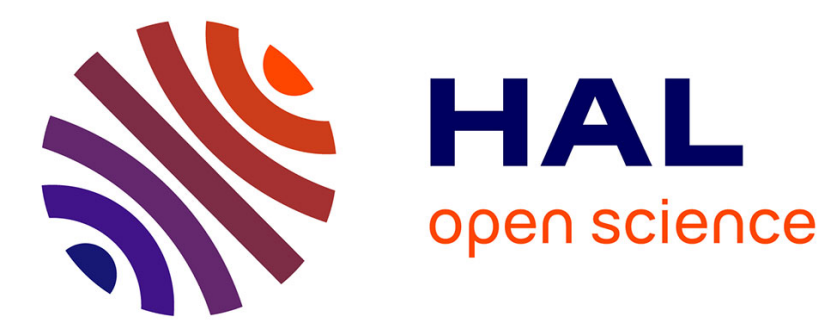

\title{
Grid spanners with low forwarding index for energy efficient networks
}

\author{
Frédéric Giroire, Stéphane Pérennes, Issam Tahiri
}

\section{To cite this version:}

Frédéric Giroire, Stéphane Pérennes, Issam Tahiri. Grid spanners with low forwarding index for energy efficient networks. Discrete Applied Mathematics, 2017, 10.1016/j.dam.2017.02.021 . hal-01634448

\author{
HAL Id: hal-01634448 \\ https://hal.inria.fr/hal-01634448
}

Submitted on 14 Nov 2017

HAL is a multi-disciplinary open access archive for the deposit and dissemination of scientific research documents, whether they are published or not. The documents may come from teaching and research institutions in France or abroad, or from public or private research centers.
L'archive ouverte pluridisciplinaire HAL, est destinée au dépôt et à la diffusion de documents scientifiques de niveau recherche, publiés ou non, émanant des établissements d'enseignement et de recherche français ou étrangers, des laboratoires publics ou privés. 


\title{
Grid spanners with low forwarding index for energy efficient networks ${ }^{\text {th }}$
}

\author{
Frédéric Giroire $^{\mathrm{a}, *}$, Stephane Perennes ${ }^{\mathrm{a}}$, Issam Tahiri ${ }^{\mathrm{b}}$ \\ ${ }^{a}$ Université Côte d'Azur, CNRS, UNS, I3S, Inria \\ Sophia Antipolis, France \\ ${ }^{b}$ Université de Bordeaux, Institut de Mathématiques, \\ UMR 5251, CNRS, Inria, 33405 Talence
}

\begin{abstract}
A routing $R$ of a connected graph $G$ is a collection that contains simple paths connecting every ordered pair of vertices in $G$. The edge-forwarding index with respect to $R$ (or simply the forwarding index with respect to $R$ ) $\pi(G, R$ ) of $G$ is the maximum number of paths in $R$ passing through any edge of $G$. The forwarding index $\pi(G)$ of $G$ is the minimum $\pi(G, R)$ over all routings $R$ 's of $G$. This parameter has been studied for different graph classes (1), (2), (3), (4). Motivated by energy efficiency, we look, for different numbers of edges, at the best spanning graphs of a square grid, namely those with a low forwarding index.

Keywords: spanning subgraphs, forwarding index, energy saving, routing, grid.

\section{Introduction}

A routing $R$ of a given connected graph $G$ of order $N$ is a collection of $N(N-1)$ simple paths connecting every ordered pair of vertices of $G$. The routing $R$ induces on every edge $e$ a load that is the number of paths going through $e$. The edge-forwarding index (or simply the forwarding index) $\pi(G, R)$ of $G$ with respect to $R$ is the maximum number of paths in $R$ passing through any edge of $G$. It corresponds to the maximum load over all edges of the graph when $R$ is used.
\end{abstract}

\footnotetext{
This work has been partially supported by ANR program ANR-11-LABX-0031-01.
}

Corresponding author. Email: frederic.giroire@cnrs.fr 
Therefore, it is important to find routings minimizing this index. The forwarding index $\pi(G)$ of $G$ is the minimum $\pi(G, R)$ over all routings $R$ 's of $G$.

The forwarding-index was introduced by Chung \& Al in 1987 (5). Due to its importance, this parameter has been studied quite extensively: on one side results have been given for different graph classes (e.g. random graphs (3), transitive and Cayley graphs $(6 ; 7)$, graphs with small numbers of vertices (2) and wellconnected graphs (4)). On the other side deep relations with other expansionrelated graph invariants have been established: Laplacian, Cheeger constant (see the survey (8)), Sparsest cut (9) and the "geometry of graphs" (10). This notion has also been used to prove that some Markov chains mix fast using either canonical paths (routings) or "resistance" (11). See the recent survey (1) for a global view on the known results.

We call a connected spanning subgraph of a graph $G$, a spanner of $G$. More precisely, it is a connected subgraph that has the same set of vertices as $G$. Our goal is to find, for a given bound on the number of edges, the best spanner of $G$, namely the one with the minimum forwarding index. The problem can also be viewed as: for a given bound $U$ on the forwarding index, find a spanner $F$ of $G$ with minimum number of edges such that $\pi(F) \leq U$.

Knowing how to solve this problem is very interesting in practice for network operators willing to reduce the energy consumed by their networks. In fact, most of the network links consume a constant energy independently of the amount of traffic they are flowing (12), (13). Therefore, it was proposed to reduce the energy used by the network links by turning some of them off, or more conveniently, putting them into an idle mode. Outside the rush hours, several studies (14), (15), (16), (17), show that a good choice of the links to turn off can lead to significant energy savings, while keeping the same communication quality. In the case where the flows from every node to every other node are of the same order, and where the capacities also lie in the same small range, a good choice of those links is reduced to the problem of finding spanners of the network with low forwarding indices.

In this paper, we consider the case in which the initial graph is a square grid. Backbone networks are generally not modeled as a grid, as showed with the typical models found in SNDLib (http://sndlib.zib.de/) and studied, e.g. in (18). However, a large number of networks are modeled by a grid in the literature. We may cite: wireless network (19), such as, wireless adhoc sensor networks (20), or random wireless networks (21), RFID reader antenna network (22; 23), mobile ad hoc networks (24), urban mesh access networks (25), femto cell networks (26), wireless backhaul networks (27), cellular networks (28), interconnection networks (29), optically interconnected arrays (30), stochastic ge- 
ometry and random graphs (31). More importantly, we wanted to understand well the difficulty of the problem on simple graphs. We thus choose to study the square grids, as they are a classical family of graphs. They are also a simple case of planar graphs. Solving the problem for square grids give hints to solve the more general case of planar graphs with bounded degrees, as they can be embedded in a grid (32). So the case of the grid is to be considered as a paradigm or a typical planar graph rather than an actual example of an existing network.

We consider the asymptotic case with $n$ large. We have two main contributions.

On one side, it is well-known that the forwarding index of the $n \times n$ grid $G_{n}$ is $\frac{n^{3}}{2}$ (see Proposition 1 (33)). An important remark is, that the load of the associated routing on the $2(n-1)^{2} \sim 2 n^{2}$ edges is lower in the corner than in the middle of the grid. Using this fact, we show how to build spanners of $G_{n}$ with much fewer edges (only $13 / 18 \approx 72 \%$ of the edges) and the same forwarding indices as $G_{n}$. We then demonstrate that our spanners are close to optimum, in the sense that we prove that it is impossible to build spanners with fewer than $4 / 3 n^{2}$ edges ( $66 \%$ of the edges).

On the other side, the smallest possible spanner of the $n \times n$ grid $G_{n}$ is a spanning tree. The forwarding index of the best spanning tree is asymptotically $\frac{3 n^{4}}{8}$, see Proposition 2 (33). When we consider spanners with a larger number of edges, the load on the edges decreases, and so does the forwarding index. In this paper, we study how the forwarding index decreases, when we increase the number of edges. The following table summarizes our results. One interesting fact is that, with $n^{2}+a^{2}$ edges (i.e $a^{2}$ extra edges), the forwarding index has order $\Theta\left(\frac{n^{4}}{a}\right)$. This is due to the planarity of the grid.

\begin{tabular}{|l||c|c|c||c|}
\hline & Spanning tree & \multicolumn{2}{c|}{ Spanners } & Grid \\
& & For an integer $a, 2 \leq a \leq n$ & \\
\hline forwarding index & $\frac{3}{8} n^{4}$ & $\frac{1}{2 a} n^{4}$ & $\frac{1}{2} n^{3}$ & $\frac{1}{2} n^{3}$ \\
\hline \hline lower bound on number of edges & $n^{2}-1$ & $\simeq n^{2}+\frac{4}{9}(0.1 a)^{2}$ & $\frac{12}{9} n^{2}$ & \\
number of edges in constructions & $n^{2}-1$ & $n^{2}+\frac{4}{9} a^{2}$ & $\frac{13}{9} n^{2}$ & $2 n^{2}$ \\
\hline
\end{tabular}

Proposition 1. (33) The forwarding index of $G_{n}$ is asymptotically $\frac{n^{3}}{2}$.

Proposition 2. (33) For $n \geq 3$, the spanning tree of $G_{n}$ with the minimum forwarding index is a tree with centroid of degree 4 and 4 branches of almost equal sizes. Its forwarding index is asymptotically $\frac{3 n^{4}}{8}$. 


\section{Spanners with the forwarding index of the grid, $\frac{n^{3}}{2}$, but much fewer edges}

In this section, we first show that a spanner with the forwarding index of the grid has at least $\frac{4 n^{2}}{3}=\frac{12 n^{2}}{9}$ edges. We then provide spanners with $\frac{13 n^{2}}{9}$ edges. But, before, we present some notations that will be used throughout the paper.

Notations. We note by $G_{n}=\left(V_{n}, E_{n}\right)$ the $n \times n$ square grid, where $V_{n}$ is the set of vertices and $E_{n}$ is the set of edges. A square grid can always be seen as $n$ rows intersecting $n$ columns. We name $v(r, c)$ the vertex at the intersection of row $r \in[n]$ with column $c \in[n]$, where $[n]$ denotes the interval of the integer numbers between 1 and $n$. An edge joining $v(r, c)$ to $v(r, c+1)$ is named $e_{h}(r, c)$ and an edge joining $v(r, c)$ to $v(r+1, c)$ is named $e_{v}(r, c)$.

Proposition 3. For any $F$ spanner of $G_{n}$ such that $\pi(F) \leq \frac{n^{3}}{2}, F$ must have, asymptotically, at least $\frac{4 n^{2}}{3}$ edges.

Proof: Consider $F$ a spanner of $G_{n}$ and let $R$ be a routing of $F$ such that $\pi(F, R) \leq$ $\frac{n^{3}}{2}$. For an integer $l \in[n]$, we call load on line $l$, the sum of the load on the edges $e_{v}(l, j) \in E(F)$, for $j \in[n]$. The load on line $l$ is $2 l(n-l) n^{2}$ as there are $l n$ vertices over line $l$ and $(n-l) n$ vertices below. If $F$ has $n-x_{l}$ edges on line $l$, there exists at least one of these edges with load at least $\frac{2 l(n-l) n^{2}}{n-x_{l}}$. As $\pi(F, R) \leq \frac{n^{3}}{2}$, we should have

$$
\frac{2 l(n-l) n^{2}}{n-x_{l}} \leq \frac{n^{3}}{2}
$$

That is

$$
n-x_{l} \geq \frac{4 l(n-l)}{n} .
$$

Thus, $F$ should have at least $\sum_{l=1}^{n} \frac{4 l(n-l)}{n}$ vertical edges. The same argument independently holds for the horizontal edges. Hence, a spanner of the grid, with load lower than $\frac{n^{3}}{2}$ on all edges, has at least

$$
2 \sum_{l=1}^{n} \frac{4 l(n-l)}{n} \text { edges. }
$$

We have

$\left.2 \sum_{l=1}^{n} \frac{4 l(n-l)}{n}=\frac{8}{n}\left(n \sum_{l=1}^{n} l-\sum_{l=1}^{n} l^{2}\right)\right)=\frac{8}{n}\left(\frac{n^{2}(n+1)}{2}-\frac{n(n+1)(2 n+1)}{6}\right)$. 
Thus, such spanner has at least

$$
\frac{4}{3}\left(n^{2}-1\right) \approx \frac{4 n^{2}}{3} \text { edges. }
$$

Theorem 1. There exists $F_{n}$ a spanner of $G_{n}$ such that $\pi\left(F_{n}\right) \sim \frac{n^{3}}{2}$ and its number of edges is asymptotically equal to $\frac{13 n^{2}}{9}$.

Proof: Let us first explain the intuition behind the construction of the spanner of the grid, $F_{n}$. We know from the proof of Proposition 3 the ratio of edges needed in every row or column in order to satisfy the lower bound. We cut the grid into small squares. Then, according to the position of the square, we use only the number of needed horizontal edges and vertical edges in each square according to the lower bound. It turns out that adding only few edges to ensure the connectivity is enough to get a spanner $F_{n}$ with a routing $R$ such that $\pi\left(F_{n}, R\right) \sim \frac{n^{3}}{2}$. The two main ingredients of the proof are that: i) Most of the load is due to "long" paths. Therefore the load due to the "end" or "start" of the paths is not significant. Similarly, the load due to local paths is not substantial. ii) The load remains approximately balanced on the vertical edges of a row (resp. horizontal edges of a column).

Construction of $F_{n}$. Let $k$ be an integer number such that $1 \ll 4 k^{3} \leq n$. Notice that this implies $k \ll \sqrt{(} n)$. We divide $G_{n}$ into small square grids of size $k \times k$. We do so by partitioning vertices of $G_{n}$ into $\left(\frac{n}{k}\right)^{2}$ sets $S_{(i, j)}$ with $i \in\left[\frac{n}{k}\right]$ and $j \in\left[\frac{n}{k}\right]: S_{(i, j)}=\left\{v(r, c) \in V_{n} ; i-1<\frac{r}{k} \leq i, j-1<\frac{c}{k} \leq j\right\}$. We call a vertex in $S_{(i, j)}$ that has a neighbor in $G_{n}$ outside $S_{(i, j)}$ a border vertex.

Let us now describe a spanner $F_{n}$ that verifies our theorem. An example of it is shown in Figure 1 in the case of $n=k^{2}=7^{2}$.

Let $t$ be the function defined on integers by $t(x)=\left\lceil 4 x k(n-x k) k / n^{2}\right\rceil$. It represents the number of needed columns (respectively rows) for a square that is on the $x$-th position horizontally (respectively vertically). We build $F_{n}$ starting from a subgraph that has all vertices of $G_{n}$ and no edges. For every $S_{(i, j)}, i, j \in$ $\left[\frac{n}{k}\right]$, we choose edges to connect vertices in $S_{(i, j)}$ in the following way:

- we add to $F_{n}$ all edges $e_{v}(r, c)$ such that $(r \bmod k) \in\{1, \ldots, t(i)\}$ (red edges in Figure 1) and 


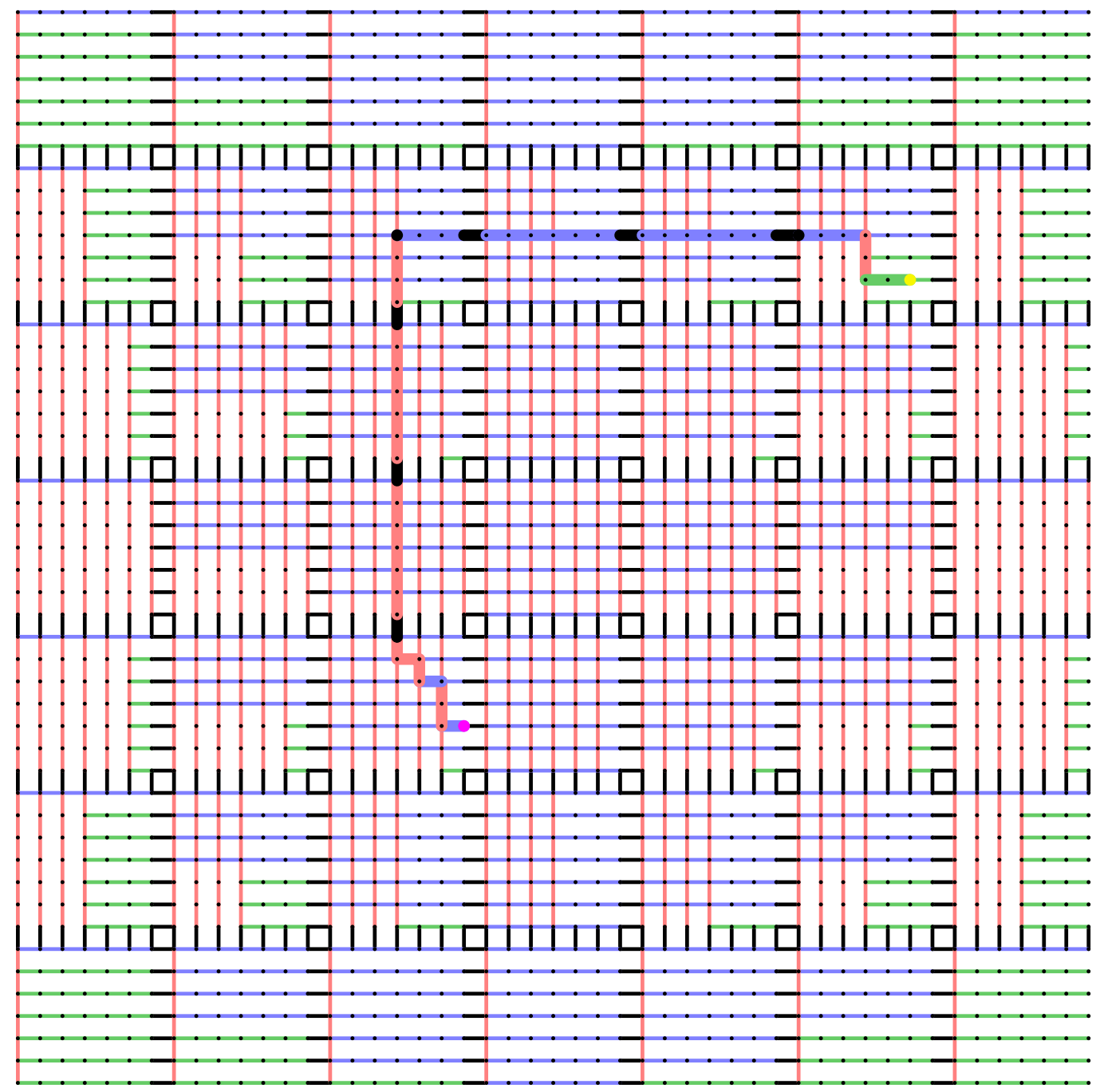

Figure 1: Construction of the spanner $F_{n}$ of Theorem 1, for $n=7^{2}$, and an example of path of the routing $R$ of $F_{n}$ (from the yellow vertex to the pink vertex).

- all edges $e_{h}(r, c)$ such that $(c \bmod k) \in\{1, \ldots, t(j)\}$ (blue edges in Figure 1);

- then we add to $F_{n}$ simple paths just to connect the remaining independent vertices (green edges in Figure 1).

- We then add all edges that do not have both endpoints in the same set $S_{(i, j)}$ 
(black edges in 1). We show in the following that adding all of them is not strictly necessary.

Description of the routing $R$. We now give a routing of the spanner $F_{n}, R$. For every ordered pair of vertices $\left(v\left(r_{a}, c_{a}\right), v\left(r_{b}, c_{b}\right)\right)$ of $V_{n}$, we describe the path connecting $v\left(r_{a}, c_{a}\right)$ to $v\left(r_{b}, c_{b}\right)$ in $R$. We distinguish two types of ordered pairs of vertices:

- Type-1 pairs: $\left\lceil r_{a} / k\right\rceil=\left\lceil r_{b} / k\right\rceil$ or $\left\lceil c_{a} / k\right\rceil=\left\lceil c_{b} / k\right\rceil$. Notice that this type includes ordered pairs with vertices that belongs to the same set $S_{(i, j)}$.

- Type-2 pairs: All the ordered pairs that do not belong to the first type.

For the Type- 1 pairs, $R$ uses the shortest path routing. For Type- 2 pairs, $R$ uses a three-segment path. An example of such path is shown in Figure 1. We name $i_{a}=\left\lceil r_{a} / k\right\rceil, i_{b}=\left\lceil r_{b} / k\right\rceil, j_{a}=\left\lceil c_{a} / k\right\rceil$ and $j_{b}=\left\lceil c_{b} / k\right\rceil$ :

- Step-1: Let $i_{m}=\min \left(i_{a}, i_{b}, n / k-i_{a}, n / k-i_{b}\right)$ and $j_{m}=\min \left(j_{a}, j_{b}, n / k-\right.$ $\left.j_{a}, n / k-j_{b}\right)$. The first segment is the shortest path from $v\left(r_{a}, c_{a}\right)$ to one of the two border vertices of $S_{\left(i_{a}, j_{a}\right)}$ that are on row $k\left(i_{a}-1\right)+t\left(j_{m}\right)$. Among the two vertices, we choose $v\left(r_{x}, c_{x}\right)$, which has the smallest distance to $S_{\left(i_{a}, j_{b}\right)}$ (as the first black vertex on the route in Figure 1).

- Step-2: Similarly, two border vertices of $S_{\left(i_{b}, j_{b}\right)}$ are on column $k\left(i_{b}-1\right)+$ $t\left(i_{m}\right)$. Among these two vertices, $v\left(r_{y}, c_{y}\right)$ is the one that has the smallest distance to $S_{\left(i_{a}, j_{b}\right)}$ (as the third black vertex on the route in Figure 1). The second segment will be linking $v\left(r_{x}, c_{x}\right)$ to $v\left(r_{y}, c_{y}\right)$ by using the path $\left[v\left(r_{x}, c_{x}\right) v\left(r_{x}, c_{y}\right) v\left(r_{y}, c_{y}\right)\right]$, which is the shortest path from $v\left(r_{x}, c_{x}\right)$ to $v\left(r_{x}, c_{y}\right)$ composed of the two direct paths $\left[v\left(r_{x}, c_{x}\right) v\left(r_{x}, c_{y}\right)\right]$, following row $r_{x}$, and $\left[v\left(r_{x}, c_{y}\right) v\left(r_{y}, c_{y}\right)\right]$, following column $c_{y}$.

- Step-3: The third and last segment will be the shortest path from $v\left(r_{y}, c_{y}\right)$ to $v\left(r_{b}, c_{b}\right)$.

Note that $k$ may be an arbitrary integer between 1 and $n$. We choose a $k$ such that $1 \ll k \ll \sqrt{n}$. For instance, we may choose $k=n^{1 / 3}$.

Number of edges of $F_{n}$. Let us compute the number of edges in the spanner, $F_{n}$. First the edges used in the subgraph induced by $S_{(i, j)}$ are all the edges on a row from 1 to $t(i)$, all edges on a column from 1 to $t(j)$, to which we add the edges 
that connect the rest of vertices through a spanning tree. Hence the number of edges in $S_{(i, j)}$ is:

$$
\begin{aligned}
& \approx t(i) \cdot k+t(j) \cdot k+(k-t(i))(k-t(j)) \\
& \approx k^{2}+t(i) t(j) \\
& \approx k^{2}\left(1+\frac{16 i j k^{2}(n-i k)(n-j k)}{n^{4}}\right) \\
& \approx k^{2}\left(1+\frac{16 i j k^{2}}{n^{2}}+\frac{16 i^{2} j^{2} k^{4}}{n^{4}}-\frac{16 i^{2} j k^{3}}{n^{3}}-\frac{16 i j^{2} k^{3}}{n^{3}}\right)
\end{aligned}
$$

The sum of those edges considering all the subsets $S_{(i, j)}$ (with $i \in\left[\frac{n}{k}\right]$ and $j \in\left[\frac{n}{k}\right]$ ) is :

$$
\begin{aligned}
& \approx k^{2} \sum_{i=1}^{n / k} \sum_{j=1}^{n / k}\left(1+\frac{16 i j k^{2}}{n^{2}}+\frac{16 i^{2} j^{2} k^{4}}{n^{4}}-\frac{16 i^{2} j k^{3}}{n^{3}}-\frac{16 i j^{2} k^{3}}{n^{3}}\right) \\
& \approx k^{2}\left[\frac{n^{2}}{k^{2}}+\frac{16 k^{2}}{n^{2}}\left(\sum_{i} i\right)^{2}+\frac{16 k^{4}}{n^{4}}\left(\sum_{i} i^{2}\right)^{2}-2 \cdot \frac{16 k^{3}}{n^{3}}\left(\sum_{i} i^{2}\right)\left(\sum_{i} i\right)\right] \\
& \approx k^{2}\left[\frac{n^{2}}{k^{2}}+\frac{16 k^{2}}{n^{2}} \cdot \frac{n^{4}}{4 k^{4}}+\frac{16 k^{4}}{n^{4}} \cdot \frac{n^{6}}{9 k^{6}}-2 \cdot \frac{16 k^{3}}{n^{3}} \cdot \frac{n^{5}}{6 k^{5}}\right] \\
& \approx k^{2}\left[\frac{n^{2}}{k^{2}}+\frac{4 n^{2}}{k^{2}}+\frac{16 n^{2}}{9 k^{2}}-\frac{32 n^{2}}{6 k^{2}}\right] \\
& =\frac{13}{9} n^{2}+o\left(n^{2}\right)
\end{aligned}
$$

The number of the remaining edges is $\approx 2 \frac{n^{2}}{k}=o\left(n^{2}\right)$, as $k \gg 1$. Therefore, as stated in the theorem, the number edges of $F_{n}$ is asymptotically equal to $\frac{13 n^{2}}{9}+o\left(n^{2}\right)$.

Load of the edges of $F_{n}$. Lets now verify that every edge has an asymptotic load which is not greater than $\frac{n^{3}}{2}+o\left(n^{3}\right)$. Consider an edge $e_{h}(r, c)$ whose incident points are in $S_{(i, j)}$. The number of Type-1 pairs that may use $e_{h}(r, c)$ is bounded by the number of pairs having one endpoint in $S_{\left(i, j_{a}\right)}$ and $S_{\left(i, j_{b}\right)}$ for some $j_{a}, j_{b} \in\left[\frac{n}{k}\right]$ and those having one end point in $S_{\left(i_{a}, j\right)}$ and $S_{\left(i_{b}, j\right)}$ for some $i_{a}, i_{b} \in\left[\frac{n}{k}\right]$. The number of these pairs is bounded by $2 k^{2} n^{2}=o\left(n^{3}\right)\left(\right.$ as $\left.k^{2}=o(n)\right)$.

Then, for Type- 2 pairs, we can start by the load induced by the segments of paths described previously in step-1 and step-3. This load is clearly bounded by the number of pairs having one endpoint inside $S_{(i, j)}$ and another endpoint 
outside $S_{(i, j)}$. The number of these pairs is bounded by: $2 k^{2}(n-k)^{2}=o\left(n^{3}\right)$ (as $\left.k^{2}=o(n)\right)$.

For Step-2, as the construction of the spanner $F_{n}$ has the needed density of edges, the average load over a line or a column is kept below $\frac{n^{3}}{2}+o\left(n^{3}\right)$ So, we only need to show that the flow is well balanced among links in $S_{(i, j)}$. Indeed, if we consider the set $L_{(i, j)}^{c}=\left\{e_{h}(r, c) \in S_{(i, j)} ; r \in\{1, \ldots, n\}\right\}$, then the total load on all these links is $\left|L_{(i, j)}^{c}\right| \frac{n^{3}}{2}+o\left(n^{3}\right)$. Thanks to the symmetries of the problem, and because, in Step-1 of the routing, we carefully choose the exit row to be $k\left(i_{a}-1\right)+t\left(j_{m}\right)$, it is enough to prove that the number of rows in $S_{(i, j)}, t(j)$ is such that $t(1)=1$ and $t(j+1)-t(j) \leq 1 \forall j \in\{1, \ldots, n /(2 k)\}$. Both of these relations hold, as we choose $k$ such that $4 k^{3} \ll n$. The same argument holds for the black edges between two adjacent subsets $S_{\left(i_{a}, j_{a}\right)}$ and $S_{\left(i_{b}, j_{b}\right)}$. This ends the proof.

\section{Spanners with forwarding indices in the range $\left[\frac{n^{3}}{2}, \frac{3 n^{4}}{8}\right]$ and Lower bounds}

We first provide spanners with forwarding indices in the range $\left[\frac{n^{3}}{2}, \frac{3 n^{4}}{8}\right]$ in Proposition 4 . We then prove that these spanners have a number of edges of the optimum order, see Proposition 5

\subsection{Spanners' constructions}

Proposition 4. Let a be an integer such that, $2 \leq a \leq n$. There exists a spanner $F_{n}(a)$ of $G_{n}$ with asymptotically $n^{2}+\frac{4}{9} a^{2}$ edges and $\pi\left(F_{n}(a)\right) \leq \frac{n^{4}}{2 a}$.

Proof: We build a spanner of $G_{n}, F_{n}(a)$, in the following way. We divide the grid into $a^{2}$ sectors. A point is in Sector $(i, j)$ if its coordinates in the grid $(\mathrm{x}, \mathrm{y})$ are such that $\frac{n}{a} i \leq x<\frac{n}{a}(i+1)$ and $\frac{n}{a} j \leq y<\frac{n}{a}(j+1)$. Each of these sectors has $(n / a)^{2}$ vertices. We call center of the sector $(i, j)$ the vertex $((i+1 / 2) n / a,(j+1 / 2) n / a)$. We consider the $a \times a$ subgrid linking all the sectors' centers. We then connect all the remaining vertices of a sector to its center with a spanning tree. This way, we get $F_{n}(a)$. Figure 2 provide a sketch of the construction of the spanner.

We now build a routing $R$ for $F_{n}(a)$. The demand between two vertices of the same sector are routed on the tree spanning their sector using the unique shortest path between them. The demand between two vertices of different sector is first routed to their centers, and then is routed in the $a \times a$ grid.

Let us compute the load of the routing $R$. We first consider the edges of the $a \times a$ subgrid. We know that an $a \times a$ grid has a routing with load $a^{3} / 2$ 


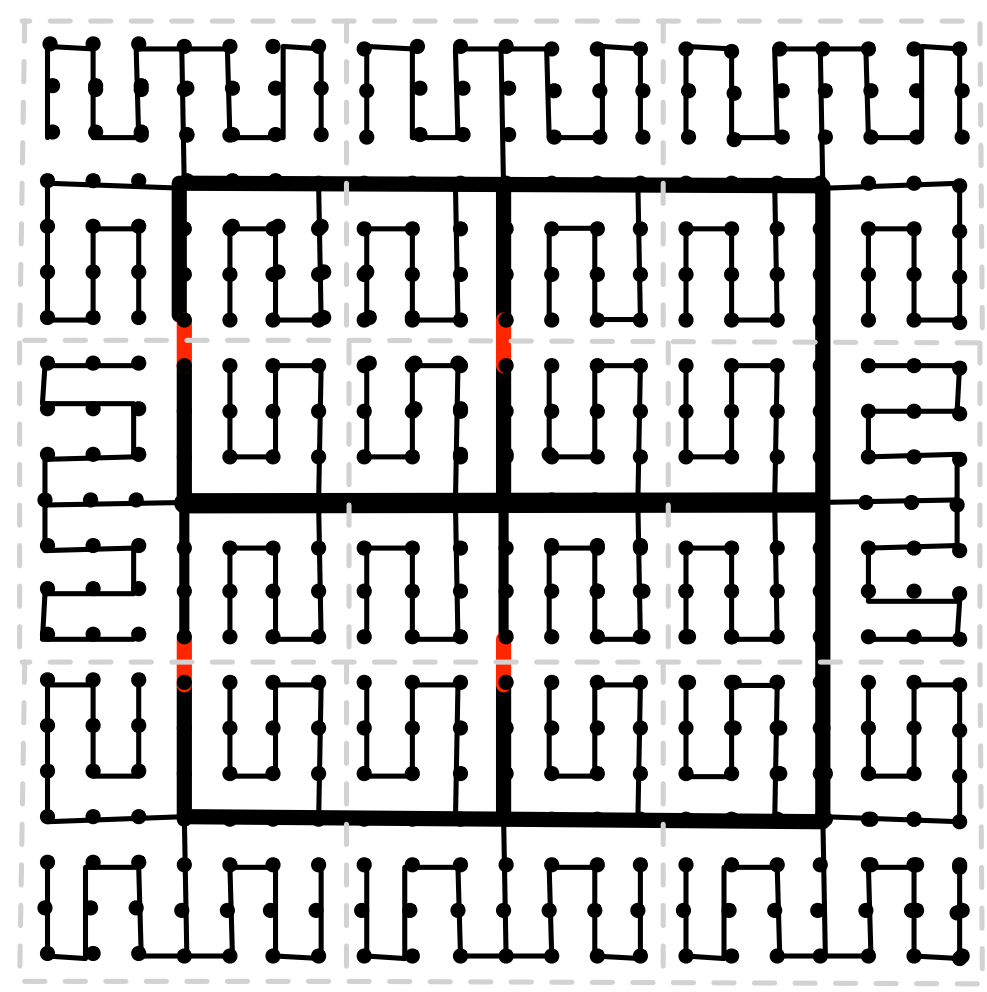

Figure 2: Spanner of Proposition 4 for $n=21$ and $a=3$. Edges of the $a \times a$ grid are in bold. Edges that are not in a spanning tree of $G_{n}$ are in red. Sectors with $(n / a)^{2}=7^{2}$ vertices are separated by dashed gray lines.

(Proposition 1). Thus, we know that it also has a $w$-routing of load $w a^{3} / 2$. Each vertex of the $a \times a$ grid receives the load of the $(n / a)^{2}$ vertices connected to it. Thus, we take $w=(n / a)^{2}$ and we obtain a $w$-routing of the $a \times a$ grid of load $\frac{a^{3}}{2}\left(\frac{n}{a}\right)^{4}=\frac{n^{4}}{2 a}$.

We then consider an edge that does not belong to the $a \times a$ grid. The only paths that can use this edge are paths going from any vertex of the grid to a vertex of its sector. Thus, its load is smaller that $(n / a)^{2} n^{2}=\frac{n^{3}}{a^{2}}$. This load is smaller than the maximum load on the $a \times a$ grid as soon as $a^{2} \geq 2 a$ which means as soon as $a \geq 2$.

Therefore $\pi\left(F_{n}(a), R\right)=\frac{n^{4}}{2 a}$.

Let us now consider the number of edges of the spanner $F_{n}(a)$. The number of 
edges necessary to connect all the nodes is $n^{2}-1$. If we choose well these edges, we just have to add $a^{2}$ edges to obtain the $a \times a$ grid (see Figure 2, additional edges are in red). $F_{n}(a)$ thus has $n^{2}+a^{2}$ edges. We can improve the spanner by using the results of Section 2 . In Theorem 1, we show that we can find a spanner of an $a \times a$ subgrid with $\frac{13}{9} a^{2}$ edges and a routing $R^{\prime}$ with the same load as a full grid with $2 a^{2}$ edges. By doing so, we get a new spanner $F_{n}(a)$, with $n^{2}+\frac{4}{9} a^{2}$ edges and $\pi\left(F_{n}, R^{\prime}\right)=\frac{n^{4}}{2 a}$.

We can rewrite the result of Proposition 4 to point out the impact of additional edges in general (Corollary 1) and when we start from a spanning tree (Corollary 2).

Corollary 1. There exist:

- A spanner of $G_{n}$ with $n^{2}+p^{2}$ edges, and an asymptotic forwarding index of $\frac{n^{4}}{3 p} \simeq 0.33 \frac{n^{4}}{p}$

- A spanner of $G_{n}$ with $n^{2}+p$ edges, and an asymptotic forwarding index of $\frac{n^{4}}{3 \sqrt{p}} \simeq 0.33 \frac{n^{4}}{\sqrt{p}}$.

Proof: Direct by Proposition 4 setting $p^{2}=\frac{4}{9} a^{2}$ or $p=\frac{4}{9} a^{2}$.

Corollary 2. There exists a spanner of forwarding index $\frac{1}{\alpha} \frac{3 n^{4}}{8}$, that is a factor $\alpha$ less than the one of the optimum spanning tree, while using $\frac{64}{81} \alpha^{2} \simeq 0.79 \alpha^{2}$ additional edges compared to a spanning tree.

Proof: Recall that an optimum spanning tree has forwarding index $\frac{3 n^{4}}{8}$, see Proposition 2. Dividing it by $\alpha$ means getting the forwarding index $\frac{3 n^{4}}{8 \alpha}=\frac{n^{4}}{2(4 \alpha / 3)}$. This is achieved by the spanner $F_{n}(a)$, with $a=4 \alpha / 3$. The spanner has an additional number of edges compared to the spanning tree equal to $\frac{4}{9}(4 \alpha / 3)^{2} \simeq 0.79 \alpha^{2}$.

\subsection{Lower bounds}

Proposition 5. There exist no spanners of $G_{n}$ with $n^{2}+p^{2}$ edges and a forwarding index less than $\frac{1}{9 \sqrt{12}} \frac{n^{4}}{p} \simeq 0.032 \frac{n^{4}}{p}$. 
Proof: Let us consider a spanner of $G_{n}$ that has $n^{2}+p^{2}$ edges. We build a multigraph in the following way. We start by assigning to every node a weight of 1 . Then, while there is still a vertex with degree 1 or 2 , we delete this vertex and the edges connecting it to the graph and divide its weight evenly among its neighbors; in case the removed vertex was of degree 2, we also connect the two neighbors afterwards. At the end of this process, we get a multigraph $H$ such that the number of its vertices $N^{\prime}$ and the number of its edges $M^{\prime}$ are related by the following equation: $N^{\prime}+p^{2}=M^{\prime}$. Indeed, every time a vertex is removed in the process leading to $H$, the number of edges is decreased by 1 . Since all the vertices in $H$ have a degree strictly greater than 2 , we have $\frac{3}{2} N^{\prime} \leq M^{\prime}$. This implies with the previous equation that $\frac{3}{2} N^{\prime} \leq N^{\prime}+p^{2}$. Hence, we have $N^{\prime} \leq 2 p^{2}$. Notice that the total weight is equal to $n^{2}$. We now apply the weighted version of the planar separator theorem (34)

on $H$ : there exists a partition of the vertices of $H$ into three subsets $A, S$, and $B$, such that each of $A$ and $B$ has at most a weight $2 n^{2} / 3, S$ has less than $\sqrt{6} \sqrt{2 p^{2}}$ vertices (The original graph is of a bounded degree 4.) and there are no edges with one endpoint in $A$ and another endpoint in $B$. This directly gives an edge cut of the original graph which has less than $2 \sqrt{6} \sqrt{2 p^{2}}$ edges and which partitions the original graph's vertices into two subsets of size at most $2 n^{2} / 3$. Therefore, any routing of this spanner will induce, at least on one edge of the cut, a load that is greater than:

$$
\frac{1}{3} n^{2} \cdot \frac{2}{3} n^{2} \cdot \frac{1}{2 \sqrt{6} \sqrt{2 p^{2}}}=\frac{1}{9 \sqrt{12}} \frac{n^{4}}{p} \simeq 0.032 \frac{n^{4}}{p}
$$

\section{Simulations and Efficiency of the Constructions}

To show that our method can be applied in practice, we compare, for a range of forwarding indices, the number of edges of the constructions proposed in Proposition 4 with the ones obtained using classical methods from the literature to find energy efficient spanners, namely an Integer Linear Program (ILP) and a heuristic algorithm (referenced as Algo) and which can be found for example in (33). The ILP takes as input a network with capacities and returns the spanner with the minimum number of edges. Algo takes the same input and removes greedily the least loaded edges as long as it is possible. 


\begin{tabular}{|c|c|ccc|}
\hline$n$ & Forwarding Index & Construction & Algo & ILP \\
\hline$a=1$ & & & & \\
\hline 4 & 128 & 15 & 15 & 15 \\
6 & 648 & 35 & 39 & $*$ \\
12 & 10368 & 143 & 168 & $*$ \\
18 & 52488 & 323 & $*$ & $*$ \\
\hline$a=2$ & & & & \\
\hline 4 & 64 & 16 & 17 & 16 \\
8 & 1024 & 64 & 64 & $*$ \\
12 & 5184 & 144 & 169 & $*$ \\
16 & 16384 & 256 & $*$ & $*$ \\
\hline$a=3$ & & & & \\
\hline 9 & 1094 & 84 & 92 & $*$ \\
12 & 3456 & 147 & 150 & $*$ \\
15 & 8438 & 228 & 230 & $*$ \\
18 & 17496 & 327 & $*$ & $*$ \\
\hline$a=4$ & & & & \\
\hline 8 & 512 & 72 & 74 & $*$ \\
12 & 2592 & 152 & 155 & $*$ \\
16 & 8192 & 264 & $*$ & $*$ \\
\hline$a=5$ & & & & \\
\hline 5 & 1000 & 115 & 116 & $*$ \\
15 & 5063 & 240 & 242 & $*$ \\
20 & 16000 & 415 & $*$ & $*$ \\
\hline
\end{tabular}

Table 1: Comparison of the number of edges in the constructions proposed in the paper, with the ones given by the heuristic algorithm Algo and an optimal ILP. An absence of values (*) means that the computation takes more than 1 hour. 
In Table 1, we give the number of edges for the spanners for different values of $a$ and $n$. We compare them with the best values found by the ILP and Algo. We used a grid of same size $n \times n$ and we set the link capacity to the forwarding index of the corresponding spanner, computed in Proposition 4 and also given in Table 1. We show that our constructions give very good results. Their number of edges is close to optimal (when it is possible to compute this value) and always better than or equal to the one given by Algo. Moreover, they are generic, structured and thus provide solutions for large networks, while the ILP and the heuristic algorithm only provide particular solutions and have a large running time (on a Quad-Core Intel Xeon $2.4 \mathrm{GHz}$ with 12 Go of RAM, the ILP cannot solve an instance of size $4 \times 4$ in one hour and Algo cannot be executed anymore for sizes larger than $16 \times 16)$.

\section{Conclusion}

In this paper we addressed the following problem: given a target bound, construct spanners of the $n \times n$ grid with a forwarding index lower than the targetbound and the smallest number of edges. We proposed spanners with a number of edges of optimum order and in some cases very close to the optimal. More precisely,

i) We provided spanners of the grid with $n^{2}+\frac{4}{9} a^{2}$ edges and forwarding indices $\frac{n^{4}}{2 a}$ (for $2 \leq a<n$ ). On the lower bound side, we proved that spanners with forwarding index $\frac{n^{4}}{2 a}$ and fewer than $\simeq n^{2}+\frac{4}{9}(0.1 a)^{2}$ edges do not exist.

ii) Similarly, we constructed spanners with $\frac{13}{9} n^{2}$ edges and with a forwarding index equal to the one of the full grid $G_{n}$. For the lower bound, we proved that spanners with such a forwarding index must have at least $\frac{12}{9} n^{2}$ edges.

Even if our constructions are quite tight, they are not optimal and this leave two open problems on the theoretical side: First, decrease the gap between our lower bound and our upper bound. Note, that we believe that closing completely this gap may be quite difficult, since it possibly implies determining a tight isoperimetric inequality for planar graphs. Second, determine if spanners with $\frac{12}{9} n^{2}$ edges and forwarding-index $\frac{n^{3}}{2}$ do exist or not.

Moreover, in this work, we focused on the square grid and aimed at providing close to optimal results. So, we studied in details a particular case of a general extremal graph theory problem, in which the goal is to find the best graph (the 
one with the fewer edges) given a bound on its forwarding index and some extra constraints (like being planar, a subgraph of the grid, ...). We believe that this extremal graph problem is interesting. However, it has not been addressed much and most of the questions are widely open (see as example (35)).

On the practical side, such spanners are important for energy efficient networks, in which the traffic has to be routed in the network, while using a minimum number of devices. The unused devices are then turned off to save energy. Note, that the case considered in the paper is the one of an all-to-all uniform traffic with homogeneous link capacities. However, the results of the paper establish useful bounds for more general settings: i) If the traffic is not all to all, the results provided in the paper (for an all-to-all traffic) give an upper bound of the number of edges needed in the spanner ii) If the capacities are not homogeneous, the results of the papers also provide an upper bound on the number of edges, if we set the capacity to the minimum link capacity of the heterogeneous case (and a lower bound if we set the capacity to the maximum link capacity). It would be interesting to investigate these more general settings in the future.

\section{References}

[1] J.-M. Xu, M. Xu, The forwarding indices of graphs-a survey, arXiv preprint arXiv:1204.2604.

[2] A. Bouabdallah, D. Sotteau, On the edge forwarding index problem for small graphs, Networks 23 (4) (1993) 249-255. doi:10.1002/net.3230230406. URL http://dx.doi.org/10.1002/net.3230230406

[3] W. Fernandez de la Vega, L. M. Gordones, The forwarding indices of random graphs, Random Structures and Algorithms 3 (1) (1992) 107-116. doi:10.1002/rsa.3240030108.

URL http://dx.doi.org/10.1002/rsa.3240030108

[4] W. F. de la Vega, Y. Manoussakis, The forwarding index of communication networks with given connectivity, Discrete Appl. Math. 37-38 (1992) 147155. doi:10.1016/0166-218X(92)90129-X.

URL http: / / dx.doi.org/10.1016/0166-218X(92) 90129-X 
[5] F. R. K. Chung, E. G. C. Jr., M. I. Reiman, B. Simon, The forwarding index of communication networks, IEEE Transactions on Information Theory 33 (2) (1987) 224-232.

[6] M. C. Heydemann, J. C. Meyer, D. Sotteau, On forwarding indices of networks, Discrete Applied Mathematics 23 (2) (1989) 103 - 123.

[7] P. Solé, Expanding and forwarding, Discrete Appl. Math. 58 (1) (1995) $67-$ 78.

[8] B. Mohar, Some applications of laplace eigenvalues of graphs, in: Graph Symmetry, Vol. 497 of NATO ASI Series, Springer Netherlands, 1997, pp. 225-275.

[9] T. Leighton, S. Rao, Multicommodity max-flow min-cut theorems and their use in designing approximation algorithms, J. ACM 46 (6) (1999) 787-832.

[10] N. Linial, E. London, Y. Rabinovich, The geometry of graphs and some of its algorithmic applications, Combinatorica 15 (1994) 577-591.

[11] A. Sinclair, Improved bounds for mixing rates of markov chains and multicommodity flow, Combinatorics, Probability and Computing 1 (1992) 351370 .

[12] J. Chabarek, J. Sommers, P. Barford, C. Estan, D. Tsiang, S. Wright, Power awareness in network design and routing, in: INFOCOM 2008. The 27th Conference on Computer Communications. IEEE, IEEE, 2008. doi:https://doi.org/10.1109/INFOCOM.2008.93.

[13] J. C. Restrepo, C. G. Gruber, C. M. Machuca, Energy profile aware routing, in: Communications Workshops, 2009. ICC Workshops 2009. IEEE International Conference on, IEEE, 2009, pp. 1-5.

[14] L. Chiaraviglio, M. Mellia, F. Neri, Reducing power consumption in backbone networks, in: IEEE International Conference on Communications, 2009, pp. 1-6. doi:10.1109/ICC.2009.5199404.

[15] R, J. Baliga, R. Ayre, K. Hinton, W, Energy consumption in IP networks, in: Optical Communication, 2008. ECOC 2008. 34th European Conference on, 2008. doi:10.1109/ECOC.2008.4729102. 
[16] F. Giroire, J. Moulierac, T. Phan, F. Roudaut, Minimization of network power consumption with redundancy elimination, in: IFIP Networking, Springer, Prague, Czech Republic, 2012, pp. 247-258.

[17] F. Giroire, J. Moulierac, T. K. Phan, Optimizing rule placement in softwaredefined networks for energy-aware routing, in: Proceedings of the IEEE Global Communications Conference (GLOBECOM), Austin, United States, 2014.

[18] F. Giroire, D. Mazauric, J. Moulierac, B. Onfroy, Minimizing routing energy consumption: from theoretical to practical results, in: IEEE/ACM International Conference on Green Computing and Communications (GreenCom'10), Hangzhou, China, 2010.

[19] M. Franceschetti, O. Dousse, N. David, P. Thiran, Closing the gap in the capacity of wireless networks via percolation theory, IEEE Transactions on Information Theory 53 (3) (2007) 1009-1018.

[20] S. Meguerdichian, F. Koushanfar, G. Qu, M. Potkonjak, Exposure in wireless ad-hoc sensor networks, in: Proceedings of the 7th annual international conference on Mobile computing and networking, ACM, 2001, pp. 139-150.

[21] R. Vaze, Random wireless networks (2015).

[22] K. Tan, A. Wasif, C. Tan, Objects tracking utilizing square grid rfid reader antenna network, Journal of Electromagnetic Waves and Applications 22 (1) (2008) 27-38.

[23] A. W. Reza, T. K. Geok, K. Dimyati, Tracking via square grid of rfid reader positioning and diffusion algorithm, Wireless Personal Communications 61 (1) (2011) 227-250.

[24] Y. Sasson, D. Cavin, A. Schiper, Probabilistic broadcast for flooding in wireless mobile ad hoc networks, in: Wireless Communications and Networking, 2003. WCNC 2003. 2003 IEEE, Vol. 2, IEEE, 2003, pp. 1124-1130.

[25] J. Camp, J. Robinson, C. Steger, E. Knightly, Measurement driven deployment of a two-tier urban mesh access network, in: Proceedings of the 4th international conference on Mobile systems, applications and services, ACM, 2006, pp. 96-109. 
[26] V. Chandrasekhar, J. G. Andrews, T. Muharemovic, Z. Shen, A. Gatherer, Power control in two-tier femtocell networks, IEEE Transactions on Wireless Communications 8 (8) (2009) 4316-4328.

[27] K. Chawla, X. Qiu, M. V. Clark, Design of a wireless backhaul network for microcells, in: Wireless Communications and Networking Conference, 1999. WCNC. 1999 IEEE, IEEE, 1999, pp. 428-432.

[28] J. G. Andrews, F. Baccelli, R. K. Ganti, A tractable approach to coverage and rate in cellular networks, IEEE Transactions on Communications 59 (11) (2011) 3122-3134.

[29] I. Stojmenovic, Honeycomb networks: Topological properties and communication algorithms, IEEE Transactions on Parallel and Distributed Systems 8 (10) (1997) 1036-1042.

[30] Z. Guo, R. G. Melhem, R. W. Hall, D. M. Chiarulli, S. P. Levitan, Pipelined communications in optically interconnected arrays, Journal of Parallel and Distributed Computing 12 (3) (1991) 269-282.

[31] M. Haenggi, J. G. Andrews, F. Baccelli, O. Dousse, M. Franceschetti, Stochastic geometry and random graphs for the analysis and design of wireless networks, IEEE Journal on Selected Areas in Communications 27 (7) (2009) 1029-1046.

[32] W. Schnyder, Embedding planar graphs on the grid, in: SoDA, Vol. 90, 1990, pp. 138-148.

[33] F. Giroire, D. Mazauric, J. Moulierac, Energy Efficient Routing by Switching-Off Network Interfaces, IGI Global, 2012, Ch. 10 - EnergyAware Systems and Networking for Sustainable Initiatives, pp. 207-236. doi:10.4018/978-1-4666-1842-8.ch010.

[34] H. N. Djidjev, On the problem of partitioning planar graphs, SIAM Journal on Algebraic Discrete Methods 3 (2) (1982) 229-240.

[35] F. Giroire, S. Pérennes, I. Tahiri, How to design graphs with low forwarding index and limited number of edges, in: 26th International Workshop on Combinatorial Algorithms (IWOCA 2015), Verona, Italy, 2015.

URL https://hal.inria.fr/hal-01221650 